\title{
Black holes with toroidal, cylindrical and planar horizons in anti-de Sitter spacetimes in general relativity and their properties ${ }^{a}$
}

\author{
José P. S. Lemos \\ Centro Multidisciplinar de Astrofísica - CENTRA, Departamento de Física, \\ Instituto Superior Técnico, \\ Av. Rovisco Pais 1, 1049-001, Lisbon, Portugal \\ E-Mail: lemos@kelvin.ist.utl.pt
}

We review the toroidal, cylindrical and planar black hole solutions in anti-de Sitter spacetimes and present their properties.

\section{Introduction}

Black holes have been objects of astrophysical interest after it was shown that they are the inevitable outcome of complete gravitational collapse of a massive star or a cluster of stars. They are likely to power the spectacular phenomena seen in x-ray emitting star accretion disks, in active galactic nuclei and quasars, and should also inhabit, in a quiescent state, the center of normal galaxies, such as our own.

Black holes appear naturally as exact solutions in general relativity. Their theoretical properties, such as their stability, the no hair theorems (stating they are characterized by three parameters only, the mass, the angular momentum and the electric charge), and the physics of matter around them, have been established. These black holes live in an asymptotically flat spacetime.

Black holes became important, not only to astrophysics, but also to physics, after the discovery that, due to quantum processes, they can emit radiation with a specific temperature, the Hawking temperature. It was also shown that they have a well defined entropy. Thus, black holes turned to be objects subject to the laws of thermodynamics.

Once they have entered the domain of physics it also became clear that one should study them not only in asymptotically flat spacetimes, but also in spacetimes with a positive cosmological constant $\Lambda>0$, i.e., asymptotically de Sitter spacetimes, and spacetimes with a negative cosmological constant $\Lambda<0$, i.e., asymptotically anti-de Sitter spacetimes. From recent astronomical observations, it seems now that we live in a world with $\Lambda>0$. However, a spacetime with $\Lambda<0$ is also worth of investigation, since it allows a consistent

${ }^{a}$ in Astronomy and Astrophysics: Recent Developments, Proceedings of the $10^{\text {th }}$ Portuguese Meeting in Astronomy and Astrophysics, Lisbon, July 2000, eds J. P. S. Lemos, A. Mourão, L. Teodoro, R. Ugoccioni (World Scientific, 2001). 
physical interpretation. Indeed, one can enlarge general relativity into a gauged extended supergravity theory, in which the vacuum state has an energy density given by $\Lambda=-3 g^{2} /(4 \pi G)$, where $g$ is the coupling constant of the theory, and $G$ is the constant of gravitation. Thus, its vacuum is described by an antide Sitter spacetime. If such types of theories are correct, it means, at least qualitatively, that anti-de Sitter should be considered as a symmetric phase of the theory, although it must have been broken, since we do not live now in a universe with $\Lambda<0$. In addition, anti-de Sitter spacetimes have other interesting features: (i) it is one of the rare gravitational backgrounds yielding a consistent interaction with massless higher spins, (ii) it permits to have a consistent theory of strings in any dimension, not only the critical ones, (iii) it has been conjectured that it has a direct correspondence with conformal field theory, the AdS-CFT conjecture, and (iv) it allows a good definition for the mass, angular momentum and charge (a property shared with the asymptotically flat case).

Most important to this review, a whole variety of classes of black holes exist in asymptotically anti-de Sitter spacetimes alone, and are amenable to be studied theoretically. Indeed, one can extended the known solutions of the Kerr-Newman family, with horizons that are spheres (in the topological sense), to include a $\Lambda<0$ term, the Kerr-Newman-anti de Sitter family (one can also extend to include a $\Lambda>0$ term, the Kerr-Newman-de Sitter family). But, for $\Lambda<0$, there are also black holes with horizons with topology different from spherical. The class we are interested her contains black holes with toroidal, cylindrical or planar topology found in 1 . Many works have been now done in this and related subjects, which also have many different connections.

\section{The solution}

The Einstein-Maxwell action is given by

$$
S=\frac{1}{16 \pi} \int d^{4} x\left(R+6 \alpha^{2}-F_{m n} F^{m n}\right),
$$

where we have put $G=c=1$ and the cosmological constant $\Lambda \equiv-3 \alpha^{2}$. A rotating solution, which can represent a black hole, may be found from the equations of motion following from (1) 3 . The metric is given by,

$$
\begin{aligned}
d s^{2}= & -\frac{\Delta}{(\alpha r)^{2}}(d t-\lambda d \phi)^{2}+\frac{(\alpha r)^{2}}{\Delta} d r^{2}+ \\
& +(\alpha r)^{2} d z^{2}+r^{2}\left(\alpha^{2} \lambda d t-d \phi\right)^{2},
\end{aligned}
$$


and the electric potential by

$$
A=-\frac{2 Q_{E}}{\alpha r}(d t-\lambda d \phi) .
$$

The Riemann $R_{k l m n}$, Ricci $R_{m n}$, and scalar $R$ curvatures can be found from (2). They yield a spacetime singularity at $r=0$. The Maxwell tensor $F_{m n}$ can be easily taken from the Maxwell curvature 2-form $F=F_{m n} d x^{m} \wedge d x^{n}$, i.e., $F=d A=\frac{2 Q_{E}}{\alpha r^{2}} d r \wedge(d t-\lambda d \phi)$, yielding two non-zero components for $F_{m n}$, $F_{r t}=2 Q_{E} /\left(\alpha r^{2}\right)$ and $F_{r \phi}=-2 Q_{E} \lambda /\left(\alpha r^{2}\right)$. A magnetic charge can easily be added.

In this solution, the ranges of the time and radial coordinates are $-\infty<$ $t<+\infty, 0 \leq r<+\infty$. The topology of the two dimensional space, $t=$ constant and $r=$ constant, can be (i) $S^{1} \times S^{1}$ the flat torus $T^{2}$ model, with $0 \leq \alpha z<2 \pi, 0 \leq \phi<2 \pi$, (ii) $R \times S^{1}$, the standard cylindrical symmetric model, with $-\infty<z<+\infty, 0 \leq \phi<2 \pi$, and (iii) $R^{2}$, the infinite plane model, with $-\infty<z<+\infty,-\infty<\phi<-\infty<$ (this planar solution does not rotate).

The functions and parameters appearing in (2) are: $\Delta \equiv 4 c^{2}-b \alpha r+\alpha^{4} r^{4}$, $\lambda \equiv \frac{1}{\alpha} \frac{\sqrt{1-\sqrt{1-\frac{8 J^{2} \alpha^{2}}{9 M^{2}}}}}{\sqrt{1+\sqrt{1-\frac{8 J^{2} \alpha^{2}}{9 M^{2}}}}}$ a specific angular momentum parameter, $J$ and $M$ being the angular momentum and mass per unit length of the system, respectively, $c^{2}=4 Q_{E}^{2}\left(1-\lambda^{2} \alpha^{2}\right)$ with $Q_{E}$ being the electric charge per unit length (for a solution with magnetic charge in addition see section 7$)$, and $b \equiv 4 M \frac{1-\lambda^{2} \alpha^{2}}{1+\frac{1}{2} \lambda^{2} \alpha^{2}}$ is a mass parameter. Note that $M=0$ implies $J=0$. For the toroidal case,

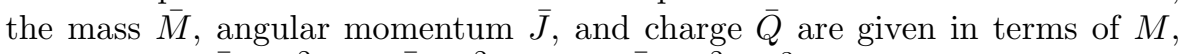
$J$ and $Q$ by $\bar{M}=\frac{2 \pi}{\alpha} M, \bar{J}=\frac{2 \pi}{\alpha} J$, and $\bar{Q}=\frac{2 \pi}{\alpha} Q$ B. When $\Delta$ has two roots the solution represents a black hole (in the toroidal model), a black string also called a cylindrical black hole (in the cylindrical model), or a black membrane also called a planar black hole (in the planar model), when it has one root it represents, correspondingly, an extreme black hole, an extreme black string, or an extreme black membrane, and when it has no roots it represents a singular closed naked line, a singular naked straight line, or a singular naked membrane, respectively.

Spatial infinity, $r \rightarrow \infty$, in these solutions has the same topology of the event horizon. This fact makes the cylindrical black hole of some astrophysical relevance, in particular, for the hoop conjecture (see below), whereas the toroidal and planar black holes seem to be of no or little astrophysical interest.

We note that the spacetime metric as given in (2) does not yield the usual form of anti-de Sitter cylindrical spacetime at spatial infinity. However, one 
can always rescale the coordinates $r$ and $z$ so that the usual form becomes apparent.

The non-rotating $J=0$, uncharged $Q=0$ black hole can be read from (2). It has a simple form

$$
d s^{2}=-\left(\alpha^{2} r^{2}-\frac{b}{\alpha^{2} r^{2}}\right) d t^{2}+\frac{d r^{2}}{\alpha^{2} r^{2}-\frac{b}{\alpha^{2} r^{2}}}+\alpha^{2} r^{2} d z^{2}+r^{2} d \phi^{2}
$$

from which the radius of the event horizon is readily taken, $r_{\mathrm{h}}=\frac{b^{1 / 3}}{\alpha}$. If we restrict the metric (4) to the toroidal case then it admits an extra parameter, the metric on the flat torus $T^{2}$ can have a more general description. Indeed, the two-manifold $t=$ constant, $r=$ constant in (4) has a metric given by $d \sigma^{2}=$ $d \theta^{2}+d \phi^{2}$, where we have put $\theta=\alpha z$. One can generalize this into the most general form of a metric on the torus, i.e., $d \sigma^{2}=|\tau|^{2} d \theta^{2}+d \phi^{2}+2(\operatorname{Re} \tau) d \theta d \phi$, where $\tau$ is the Teichmüler complex parameter on the torus giving classes of conformally equivalent tori $(\operatorname{Im} \tau>0)$. This parameter has several implications on the structure of the black holes, for instance, the size of the event horizon depends on the conformal class of the torus $1, r_{\mathrm{h}}=\frac{2}{\pi} \frac{\bar{M}}{\alpha^{2}|\operatorname{Im} \tau|}$.

In terms of the $\lambda^{2} \alpha^{2}$ angular-momentum, charge $Q$, and mass $M$ one can distinguish clearly five zones: (i) $0 \leq \lambda^{2} \alpha^{2}<1-\frac{64 Q^{6}}{27 M^{4}}\left(1+\alpha^{2} \lambda^{2}\right)^{4}$ : black hole region, (ii) $\lambda^{2} \alpha^{2}=1-\frac{64 Q^{6}}{27 M^{4}}\left(1+\alpha^{2} \lambda^{2}\right)^{4}$ : extreme black hole line, (iii) $1-\frac{64 Q^{6}}{27 M^{4}}\left(1+\alpha^{2} \lambda^{2}\right)^{4}<\lambda^{2} \alpha^{2}<1$ : naked singularity region, (iv) $\lambda^{2} \alpha^{2}=1$ : null singularity line, (v) $1<\lambda^{2} \alpha^{2} \leq 2$ : pathological black hole region (black holes with closed timelike curves). For $J>\sqrt{\frac{9 M}{3 \alpha}}$, the specific angular momentum parameter $\lambda$ turns complex, and the metric is ill-defined. The extreme case is given when $Q$ is connected to $\lambda$ through the relation $Q^{6}=\frac{27}{64} \frac{1-\lambda^{2} \alpha^{2}}{1+\frac{1}{2} \lambda^{2} \alpha^{2}}$. For the Penrose diagrams see 3 . In the uncharged case, one has three zones only 2 , (i) $0 \leq \lambda^{2} \alpha^{2}<1$ : black hole region, (ii) $\lambda^{2} \alpha^{2}=1$ : extreme black hole line, and (iii) $1<\lambda^{2} \alpha^{2} \leq 2$ : naked singularity region. The metric with electric charge andzero angular momentum was also discussed in 5 . For the Penrose diagrams seet.

The black holes as well as the naked singularities can appear through gravitational collapse, the black holes display quasi-normal modes, have interesting properties due to their horizon topology, the non-rotating configurations have thermodynamical properties related to Reissner-Nordström black holes, some of these black holes are supersymmetric, and there are many connections with other black hole solutions, as will be shown in the next sections. 


\section{Gravitational collapse}

From the work of Oppenheimer and Snyder 8 and Penrose's theorem 8 we know that if general relativity is correct, then realistic, slightly non-spherical, complete collapse leads to the formation of a black hole and a singularity. This result tights strongly the theory of gravitational collapse to the theory of black holes. In this connection, two important conjectures were formulated, the cosmic censorship and the hoop conjectures. The cosmic censorship conjecture 9 forbids the existence of naked singularities, singularities not surrounded by an event horizon of a black hole. The hoop conjecture 10 states that black holes form when and only when a mass $M$ gets compacted into a region whose circumference in every direction is less than its Schwarzschild circumference $4 \pi M(G=c=1)$.

Thus, motivated by the cosmic censorship and hoop conjectures, it is important to understand, besides spherical collapse, highly non-spherical collapse. The collapses of prolate spheroids, such as spindles, or oblate spheroids, such as pancakes, are not only astrophysically interesting but also important to a better understanding of these conjectures. Prolate collapse has been studied in some detail 11 and it was shown that fully relativistic effects, totally different from the spherical case, come into play. Ipaddition, cylindrical systems were used by Thorne to mimic prolate collapse $₫$. Oblate collapse has been mainly studied within Newtonian theory, in connection with galaxy formation, but relativistic results are also available.

All these results were applied to spacetimes with zero cosmological constant. In the presence of a negative cosmological constant one can expect the occurrence of major changes. Indeed, we showed in section 2 that there are black hole solutions with toroidal, cylindrical or planar topology if a negative cosmological constant is present. Since the importance of the spherical Schwarzschild black hole has first come from its role as the final state of complete gravitational collapse of a star, it is useful to investigate if these black holes with different topology may also emerge from gravitational collapse of some matter distribution. Moreover, one would like to test the cosmic censorship and hoop conjectures in these cases.

An important feature of spherical collapse onto a Schwarzschild black hole is that, due to Birkhoff's theorem, spacetime is static outside the matter and the collapse proceeds without emission of gravitational waves. The same is true for the collapse of spherical matter in an anti-de Sitter background. On the other hand, it is well known that the collapse of cylindrical systems proceeds with emission of gravitational waves which creates additional problems in their modeling. However, surprisingly, the problem can be solved exactly 
12 by using, for the exterior spacetime, a modified Vaidya metric appropriate to the cylindrical collapse (we refer here to the cylindrical case, only because it is the most interesting for gravitational collapse and for the above conjectures, although the results apply equally well to toroidal an planar collapses). This modified Vaidya metric describes the gravitational field associated with a cylindrical flow of unpolarized scalar, neutrino, electromagnetic or gravitational radiation in the geometrical optics approximation. The interior solution, is a modified Friedmann solution also applicable to cylindrical topology. Through a smooth matching at the interface, we find that the flux of waves modeled by the modified Vaidya metric is an incoming flux, and consequently that the mass parameter of the collapsing matter grows up to the formation of the black hole. By carefully choosing the right amount of incoming flux one avoids the emission of gravitational waves from the collapsing matter. One can then show that that a black hole forms, and study the physics from the inside and outside points of view. It has much the same characteristics of the formation of a Schwarzschild black hole 12 .

It is also possible to test the cosmic censorship and hoop conjectures $13 \mathrm{fpr}$ collapse in a background with a negative cosmological constant. It was found 13 that (i) in spherical collapse with negative $\Lambda$, massless naked singularities form for a sufficiently inhomogeneous collapse, similarly to the $\Lambda=0$ case, and (ii) cylindrical (planar or toroidal) collapse with negative $\Lambda$ does not produce naked singularities, in accordance with the cosmic censorship; instead black strings (black membranes or black holes, respectively) will form giving an explicit counter-example to the hoop conjecture. For charged collapsing matter naked singularities form, violating the cosmic censorship 4 .

To theoretical astrophysics the interesting black hole is the one with cylindrical topology although an object with cylindrical topology (and symmetry) in an anti-de Sitter cylindrical spacetime is an idealized situation, of course. However, it is possible that the universe we live in contains an infinite cosmic string. It is also possible, that the universe is crossed by an infinite black string. The solution with planar topology, a black membrane, can be of some relevance. On the other hand, the toroidal topology does not seem to be of immediate interest to astrophysics. The reason is that the flat torus is constructed from multiplying two circles. Since each circle lives in a two dimensional plane, the flat torus could only be visualized inside a four dimensional Euclidean (flat) space, not in a three dimensional one like ours. However, if the universe has some exotic topology at infinity it could accommodate a flat torus black hole (see section on the topology of the horizon below). A flat torus black hole can also be of relevance to theories of elementary particles invoking extra dimensions. 


\section{Quasi-normal modes}

Perturbations of stars and black holes reveal much of their intrinsic structure. For compact objects such as neutron stars and black holes the study of perturbations is closely linked to the gravitational wave emission and is therefore relevant to gravitational wave astronomy. Much work has been done in asymptotically flat spacetimes 15 . It is thus also important to study the properties under perturbations of the black holes that live in anti-de Sitter spacetimes and test for their stability.

Some objects that we are used to perturb, for instance a string, display a whole set of normal modes (with real frequencies) which give interesting and important properties. For gravitational objects, such as a black hole, as the system starts to vibrate it emits gravitational waves and damps down. In this case, due to the emission of gravitational waves, normal modes do not exist. Instead, there are quasi-normal modes with frequencies that have real parts (signalling the vibration of the hole) and imaginary parts (representing the damping down). It is, of course, important that the imaginary part of the frequency yields a damping mode (not a growing one) such that the whole perturbation is stable. This is the case for the spherical horizon of the Schwarzschild black hole 15 . It is also the case for merturbations of spherical black holes in anti-de Sitter spacetimes, seet and 17 for references, as well as for toroidal, cylindrical and planar black holes 18. The emission of waves from aparticle falling down into an anti-de Sitter black hole can also be considered 19.

\section{The Topology of the Horizon}

The topology of the horizon of the black holes we have been presenting can be toroidal, cylindrical and planar. However, it was shown by Hawking 20 as a theorem, that under certain reasonable physical conditions (spacetime is asymptotically flat and globally hyperbolic, and the dominant energy condition holds), the topology of the event horizon is spherical. This result was hinted by the topology of the known black holes, the Schwarzschild and Kerr black holes, and their charged generalizations, where the shape of the event horizon is spherical (or easily molded into a spherical shape) Thus it came as a surprise, that the black holes we have discussed found in 4 , with topologies other than spherical are solutions of Einstein's equations. The reason that they are not in accord with Hawking's theorem is that, due to the presence of a negative cosmological constant, spacetime is neither asymptotically flat nor globally hyperbolic. In order to accommodate these topologies into general relativity one has to generalize Hawking's theorem. This can be done by using 
the topological censorship theorem 21, which states that the topology of spacetime cannot be directly probed by distant observers. Using this idea, one can generalize the theorem valid for asymptotically flat spacetimes and show that there is a relationship between the topology of the horizon and the topology at infinity 22 (see 23 also). It is then possible to have toroidal, cylindrical or planar horizons in a toroidal, cylindrical or planar spacetimes at infinity.

\section{Thermodynamic properties}

We now turn to quantum aspects. We first study the thermodynamical properties of these black holes and compare them to their spherically symmetric counterparts. One direct approach to thermodynamics is through the path-integral approach. Its application to the thermodynamics of black holes was originally developed by Hawking et al. 24-26. In this approach the thermodynamical partition function is computed from the path-integral in the saddle-point approximation, allowing one to obtain the thermodynamical laws for black holes. In the path-integral approach we can use the three different ensembles: microcanonical, canonical and grand canonical. Due to difficulties related to stability of the black hole in the canonical ensemble, the microcanonical ensemble was originally considered 26. However, further developments by York et al. 27 28 allowed to define the canonical ensemble. Effectively, by carefully defining the boundary conditions, one can obtain the partition function of a black hole in thermodynamical equilibrium. This approach was further developed to include other ensembles, and to study charged black holes in the grand canonical ensemble and black holes in asymptotically anti-de Sitter spacetimes 29.30 . In York's formalism the black hole is enclosed in a cavity with a finite radius. The boundary conditions are defined according to the thermodynamical ensemble under study. Given the boundary conditions and imposing the appropriate constraints, one can compute a reduced action suitable for doing black hole thermodynamics. Evaluating this reduced action at its stable stationary point one obtains the corresponding classical action, which is related to a thermodynamical potential. In the canonical ensemble this thermodynamical potential corresponds to the Helmholtz free energy, while for the grand canonical ensemble the thermodynamical potential is the grand canonical potential. From the thermodynamical potential one can compute all the relevant thermodynamical quantities and relations.

In order to compare the thermodynamics of charged black holes with spherical horizons 30 and charged black holes with toroidal horizons 31 we have used York's formalism in the grand canonical ensemble. An important quantity is the entropy, which for toroidal black holes is given by $S=\pi^{2} r_{+}^{2}$. where $r_{+}$ 
is the horizon radius of the charged black hole. Since $\pi^{2} r_{+}^{2}=A_{+} / 4$, where $A_{+}$is the area of the event horizon, we have $S=\frac{A_{+}}{4}$. This is the usual Hawking-Bekenstein entropy, which means this law is still valid for black holes with toroidal symmetry. Another important quantity is the heat capacity $C$ of the black hole. For a simple case, when the boundary of the cavity goes to infinity one gets 31, $C=2 \pi^{2} r_{+}^{2}\left(1-\frac{2 Q_{E}^{2}}{Q_{E}^{2}+3 \pi^{2} \alpha^{2} r_{+}^{4}}\right)$. One can compute more general cases for instance when the boundary of the cavity is held at a finite value $r_{B} 29$. B1. The heat capacity in this case is always positive which means these solutions are all stable. Furthermore we find that in the grand canonical ensemble, with temperature and electrostatic potential fixed at the boundary, there is a black hole solution that is globally stable, which means it dominates the grand partition function. These results are generally different from the results obtained for the spherical counterpart of this black hole, the Reissner-Nordström-anti-de Sitter black hole, for which there are one or two solutions, that can be stable or unstable, and do not necessarily dominate the grand partition function 30 . Thus, contrary to the Reissner-Nordström-anti de Sitter black hole, for the toroidal black hole no phase transition was found.

For other studies of thermodynamic properties of these black hgles see 632 , and for the subtle issue of the entropy of extreme black holes see 3 .

\section{Supersymmetries}

To go beyond the astrophysical properties of the black holes, such as the issue of gravitational collapse, and try to understand a black hole as a particle, a massive elementary particle, it is also important to embed these objects in some comprehensive particle theory, beyond general relativity, such as a supergravity theory. Black holes in these theories are called supersymmetric black holes. There are several motivations to, first, find supersymmetric black hole solutions and, then, analyze their properties. For instance, black holes that are supersymmetric, have zero temperature, and in some cases it is possible to associate them with solitons of the underlying theory, interpolating between two distinct homogeneous vacua solutions. In addition, in certain instances, supersymmetric black holes are exact solutions of the theory even when quantum corrections are taken into account.

There are by now many supersymmetric black hole solutions in many different supergravity theories. A characteristic of supersymmetric theories is that there are as many bosons as fermions. We are interested in a particular theory, called $N=2$ gauged supergravity, since it yields the Einstein-Maxwell theory with a negative cosmological constant, when there are no fermions. One can then look for the black hole solutions we have found in section 2, which 
remain the same when one makes the allowed symmetry transformations of the underlying theory. These black hole solutions are the supersymmetric black holes. Normally, since one is treating a theory of elementary particles, one also includes in the solutions magnetic charge in addition to electric charge.

The supersymmetry of the spherical solutions, such as the Schwarzschild anti-de Sitter solution, studied in 3435 show that to be supersymmetric the black hole must rotate. Subsequent studies for other types of black holes (see section 8.1) were performed in 36.37 , with additional features not present in the spherical case.

For the black hole presented in section 2, one can also study its supersymmetry properties 38 . The study of the integrability conditions has shown that the supersymmetric black hole has zero electric and magnetic charge, must rotate with extreme angular velocity and thus has zero temperature. The corresponding Killing spinor is $\epsilon=\sqrt{r}\left[\frac{1}{2}\left(1-\gamma_{1}\right)\right] \epsilon_{0}$, where $\gamma_{1}$ is a Dirac matrix in the real representation, and $\epsilon_{0}$ is a constant Dirac spinor. Due to the projection operator $\frac{1}{2}\left(1-\gamma_{1}\right)$, this equation defines two linearly independent Dirac spinors. Thus, this extreme uncharged black hole has two supersymmetries, the same number as the background spacetime. There are other non-rotating solutions, representing charged naked singularities, which are supersymmetric. For further details see 38 .

\section{Connections to other black holes}

\subsection{Non-isometric toroidal solutions and multi-tori solutions}

The rotating solution (equations (2)-(3)) can be obtained from the static toroidal metric by mixing time and angle into a new time and a new angle. Since angles are periodic, this is not a proper coordinate transformation, yielding a new solution globally different from the static one (seel).

A different rotating toroidal metric can be extracted from the general Petrov type-D solution by choosing the corresponding parameters in an appropriate way and imposing time-angle symmetry $t \rightarrow-t$ and $\phi \rightarrow-\phi 39$. This metric cannot be obtained by the above coordinate mixing, and thus, it implies that flat torus topology for the $r=$ constant, $t=$ constant surfaces admits at least two distinct rotating black hole solutions not isometric to each other, although when the rotation parameter is zero they are isometric.

A toroidal black hole has a smooth orifice (non-black) in its middle, so to speak. The event horizon is a Riemann surface with genus $g=1$. There are other solutions with more than one orifice through the horizon, i.e., black hole solutions with higher genus, $g>1$. These other types of topological black holes were found in 4042 and their properties studied. Black holes with exotic 
horizons also appear in other theories, such has dilatonic theories derived from the low-energy actions of string theory $43,44,4$. We do not dwell on these very interesting topics here.

\section{2 (2+1)- and (1+1)-dimensional black holes}

The black holes in 3+1-dimensions, i.e., in four-dimensions $(4 \mathrm{D})$, presented in section 2 , are also solutions in $(2+1)$-dimensions (3D) 2 and in $(1+1)$ dimensions (2D) 1 . In fact, since the $z$ and $\phi$ directions are Killing directions one can perform a dimensional reduction in one direction, say the $z$ direction to get a 3D black hole, and in both $z$ and $\phi$ directions to get a $2 \mathrm{D}$ black hole.

By dimensionally reducing the action (11) through one dimension one gets the following 3D action, $S=\int d^{3} x \sqrt{-g} e^{-2 \phi}\left(R-4 \omega(\partial \phi)^{2}+4 \lambda^{2}\right)$, where $g$ is the determinant of the $3 \mathrm{D}$ metric, $R$ is the $3 \mathrm{D}$ curvature scalar, $\phi$ is a scalar called the dilaton field, which has come from the extra dimension, $\lambda$ is a constant (related to $\Lambda$ ), and $\omega=0$ in this reducing process, but can be made a free parameter in general. This is a Brans-Dicke action in 3D. Now, each different $\omega$ can be viewed as yielding a different dilaton gravity theory. For instance, for $\omega=0$ it yields the above theory, equivalent in certain ways to $4 \mathrm{D}$ oeneral relativity $\mathrm{l}$; for $\omega=-1$ one gets the simplest low energy string action 46; for $\omega=\infty$ one obtains 3D general relativity and its BTZ black hole 474 (see 19 for perturbation on this black hole and references therein, see 50 for multi-black hole solutions). Each different $\omega$ has a very rich and non-trivial structure of solutions with many black holes, which can be considered on its own. Here we do notattempt to comment on the whole set of solutions-gethe different theories 51.52 . For electric and magnetic rotating solutions see 56.57 .

Analogously, one gets on reduction the action (17) through two dimensions the following 2D action, $S=\int d^{2} x \sqrt{-g} e^{-2 \phi}\left(R-4 \omega(\partial \phi)^{2}+4 \lambda^{2}\right)$, where now $\omega=-\frac{1}{2}$ in this reducing process, but can be made again a free parameter in general. Thus $\omega=-\frac{1}{2}$ yields the above $2 \mathrm{D}$ theory, equivalent in certain ways to $4 \mathrm{D}$ general relativity $\mathrm{\omega}$; for $\omega=-1$ one gets the simplest low energy string action 46 ; for $\omega=\infty$ one obtains the two dimensional analogue of general relativity 53 We refer to the appropriate papers and citations in the following papers $54-56$

\subsection{Higher-dimensional black holes}

Higher dimensional black holes appear in several gravity theories and some can have an interpretation in 4D upon a suitable Kaluza-Klein dimensional reduction (see 58 for a review in higher and lower dimensions). Black holes in 
dimensions higher than 4 in anti-de Sitter backgrounds have been found 5960 . All these black holes, in low, in 4, and in higher dimensions are important in connection to the AdS/CFT conjecture. This conjectures states a correspondence between supergravity (the low energy limit of string theory) on an anti-de Sitter space and a conformal field theory on the boundary of that space 6162 . For instance, due to this AdS/CFT duality, quasi-normal frequencies in the BTZ black hole spacetime yield a prediction for the thermalization timescale in the dual two-dimensional conformal field theory, which otherwise would be very difficult to compute directly. If one has, e.g., a 10-dimensional type IIB supergravity, compactified into a BTZ $\times S^{3} \times T^{4}$ spacetime, the scalar field used to perturb the BTZ black hole, can be seen as a type IIB dilaton which couples to a conformal field theory operator $\mathcal{O}$. Now, the BTZ in the bulk corresponds to a thermal sate in the boundary conformal field theory, and thus the bulk scalar perturbation corresponds directly to a thermal perturbation with nonzero $\langle\mathcal{O}\rangle$ in the conformal field theory. Similar arguments related to black hole thermodynamics in 4 and higher dimensions also hold 5962 .

\section{Conclusions}

We have reviewed the toroidal, cylindrical and planar black holes that appear as solutions of general relativity with a negative cosmological constant and study some of their properties. Other properties that could be studied are the uncommon behavior of the luminosity 63 , the propagation of vacuum polarized photons 64, and black hole pair production (see 65.66 and references therein) to name three.

What are the uses of these black holes?

In astrophysics, as we know it, little, apart some better understanding of the hoop conjecture: formation of cylindrical black holes in anti-de Sitter spacetimes provide a direct counter-example to the conjecture.

For general relativity, they yield new exact solutions that should be added to the Kerr-Newman family. The existence of topological black holes enlarges the field of geometry in which general relativity and related theories act. The beautiful pure geometric constructions of the BTZ 3D black hole 48.5 and of the constant curvature $4 \mathrm{D}$ black holes 40 are there to prove it.

In theories of elementary particles dealing with Planck scales, such as string theory with its elementary strings and branes, a black hole is itself considered as a phase state of elementary strings with about one Planck mass obeying the laws of quantum mechanics. In such theories all types of black holes in any permitted dimension must be important. These black holes we have been discussing are certainly important just above the Planck scales, where there 
are indications that the vacuum is anti-de Sitter. They might be detected one day through gravitational radiation, Hawking evaporation, or black hole pair production. In some instances_one can now calculate from first principles the black hole entropy 67 (see 66.69. 60 for reviews). This leads to arguments in favor of the resolution of the information paradox for certain black holes. However, topological hlack holes in anti-de Sitter spacetimes do not fit easily into those arguments 36,39. As a last use, black holes in anti-de Sitter spaces are at the center of the AdS/CFT conjecture mentioned above.

The asymptotic anti-de Sitter black holes, like the asymptotically flat ones, can appear in any scale from the Planck size to astrophysical dimensions. This property of black holes is unique in a sense, all other objects, either have a well defined scale, or a small range of scales (for a review on the sizes of black holes see 71 ).

We have tried to quote all the literature relevant and somehow connected to this subject. This was intended to be a six pages review, it has grown to more than the double, and it is now clear that the subject of topological black holes, not only the ones mentioned here, deserve a thorough review.

\section{Acknowledgments}

I thank Observatório Nacional - Rio de Janeiro for hospitality, while part of this work was being written. This work was partially funded by FCT - Fundação para a Ciência e Tecnologia through project ESO/PRO/1250/98. 


\section{References}

1. J.P.S. Lemos, Class. Quantum Grav. 12, 1081 (1995).

2. J.P.S. Lemos, Phys. Lett. B 353, 46 (1995).

3. J.P.S. Lemos, V.T. Zanchin, Phys. Rev. D 54, 3840 (1996).

4. J.P.S. Lemos, V.T. Zanchin, hep-th/9511188.

5. C.G. Huang, C-B. Liang, Phys. Lett. A 201, 27 (1995).

6. L. Vanzo, Phys. Rev. D 56, 6475 (1997).

7. J.R. Oppenheimer, H. Snyder, Phys. Rev. 56, 455 (1939).

8. R. Penrose, Phys. Rev. Lett. 14, 57 (1965).

9. R. Penrose, Riv. Nuovo Cimento (Numero Special) 1, 252 (1969).

10. K.S. Thorne in in Magic without Magic, ed. J. R. Klauder (Freeman and Company, 1972), p. 231.

11. F. Echeverria, Phys. Rev. D 47, 2271 (1993).

12. J.P.S. Lemos, Phys. Rev. D 57, 4600 (1998).

13. J.P.S. Lemos, Phys. Rev. D 59, 044020 (1999).

14. S.G. Ghosh, Phys. Rev. D 62, 127505 (2000).

15. K.D. Kokkotas, B.G. Schmidt, http://www.livingreviews.org/Articles/ Volume2/1999-2kokkotas/; gr-qc/9909058.

16. G.T. Horowitz, V.E. Hubeny, Phys. Rev. D 62, 024027 (2000).

17. V. Cardoso, J.P.S. Lemos, Phys. Rev. D, in press (2001); gr-qc/0105103 (see also the corresponding paper in this volume).

18. V. Cardoso, J.P.S. Lemos, "Quasi-normal modes of toroidal and cylindrical black holes in anti de Sitter spacetimes", in preparation, (see also gr-qc archives, to be submitted, 2001).

19. V. Cardoso, J.P.S. Lemos, "Radiation from particles falling into Schwarzschild-anti de Sitter black holes", in preparation, (see also grqc archives, to be submitted, 2001).

20. S.W. Hawking, Comm. Math. Phys. 25, 152 (1972); S.W. Hawking, G.F.R. Ellis, The Large scale structure of spacetime, (Cambridge University Press, 1973), p. 373.

21. J.L. Friedman, K. Schleich, D.M. Witt, Phys. Rev. D 71, 1486 (1993) ; Erratum: 75, 1872, (1995).

22. G.J. Galloway, K. Schleich, D.M. Witt, E. Woolgar, Phys. Rev. D 60, 104039 (1999).

23. T. Jacobson, S. Venkataramani, Class. Quantum Grav. 12, 1055 (1995).

24. J.B. Hartle, S.W. Hawking, Phys. Rev. D 13, 2188 (1976).

25. G.W. Gibbons, S.W. Hawking, Phys. Rev. D 15, 2752 (1977).

26. S.W. Hawking, in General Relativity: An Einstein Centenary Survey, ed. S.W. Hawking, W. Israel (Cambridge University Press, 1979). 
27. J.W. York, Phys. Rev. D 33, 2091 (1986).

28. J.D. Brown, J.W. York, in The Black Hole 25 Years After, ed. C. Teitelboim and J. Zanelli (Plenum, New York 2000); gr-qc/9405024.

29. D.R. Brill, J. Louko, P. Peldán, Phys. Rev. D 65, 3600 (1997).

30. C. Peça, J.P.S. Lemos, Phys. Rev. D 59, 124007 (1999).

31. C. Peça, J.P.S. Lemos, J. Math. Physics 41, 4783 (2000).

32. A. Chamblin, R. Emparan, C.V. Johnson, R.C. Myers, Phys. Rev. D 60, 104026 (1999).

33. A. Ghosh, P. Mitra, Phys. Rev. Lett. 78, 1858 (1997).

34. L.J. Romans, Nucl. Phys. B 383, 395 (1992).

35. V.A. Kostelecký, M.J. Perry, Phys. Lett. B 371, 191 (1996).

36. M.M. Caldarelli, D. Klemm, Nucl. Phys. B 454, 434 (1999).

37. N. Alonso-Alerca, P. Meessen, T. Ortín, Class. Quantum Grav. 17, 2783 (2000).

38. J.P.S. Lemos, Nucl. Phys. B 600, 272 (2001).

39. D. Klemm, V. Moretti, L. Vanzo, Phys. Rev. D 57, 6127 (1998).

40. S. Åminneborg, I. Bengtsson, S. Holst, P. Peldán, Class. Quantum Grav. 13, 2707 (1996).

41. R.B. Mann, Class. Class. Quantum Grav. 14, L109 (1997).

42. W.L. Smith, R.B. Mann, Phys. Rev. D 56, 4942 (1997).

43. G.T. Horowitz, "The Dark Side of String Theory", hep-th/9210119.

44. R.G. Cai, Y.Z. Zhang, Phys. Rev. D 54, 4891 (1996).

45. R.G. Cai, K.S. Soh, Phys. Rev. D 59, 044013 (1999).

46. C. Callan, D. Friedan, E. Martinec and M. Perry, Nucl. Phys. B 262, 593 (1985).

47. M. Bañados, C. Teitelboim and J. Zanelli, Phys. Rev. Lett. 69, 1848 (1992).

48. M. Bañados, M. Henneaux, C. Teitelboim and J. Zanelli, Phys. Rev. D 48, 1506 (1993).

49. V. Cardoso, J.P.S. Lemos, Phys. Rev. D 63, 124015 (2001).

50. D. Brill, Annalen Phys. 9, 217 (2000).

51. P.M. Sá, A. Kleber, J.P.S. Lemos, Class. Quantum Grav. 13, 125 (1996).

52. P.M. Sá, J.P.S. Lemos, Phys. Lett. B 423, 49 (1998).

53. J.P.S. Lemos, P.M. Sá, Phys. Rev. D 49, 2897 (1994).

54. J.P.S. Lemos, P.M. Sá, Class. Quantum Gravity 11, L11 (1994).

55. J.P.S. Lemos, P.M. Sá, Mod. Phys. Lett. A 9, 771 (1994).

56. O.J.C. Dias, J.P.S. Lemos, Phys. Rev D, in press (2001), hep-th/0105183.

57. O.J.C. Dias, J.P.S. Lemos, "Static and rotating magnetic black holes in three-dimensional Brans-Dicke gravity theories ", in preparation, (see also hep-th archives, to be submitted, 2001). 
58. J.P.S. Lemos, "A Profusion of Black Holes from Two to Ten Dimensions", Proceedings of the XVII $\underline{t}^{h}$ Encontro Nacional de Física de Partículas e Campos, in commemoration of the $30^{\text {th }}$ anniversary of the Sociedade Brasileira de Física (SBF), ed. A. J. Silva, (USP, 1997), p. 40; hepth/9701121.

59. D. Birmingham, Class. Quantum Grav. 16, 1197 (1999).

60. D. Klemm, JHEP 0102, 031 (2001).

61. J.M. Maldacena, Adv. Theor. Math. Phys. 2, 253 (1998).

62. E. Witten, Adv. Theor. Math. Phys. 2, 505 (1998).

63. D. Klemm, L. Vanzo, Phys. Rev. D 58, 104025 (1998).

64. R.G. Cai, Nucl. Phys. B 524, 639 (1998).

65. O.J.C. Dias, see paper in this volume; gr-qc/0106081.

66. O.J.C. Dias, J.P.S. Lemos, J. Math. Phys., in press (2001), hep$\mathrm{ph} / 0103193$.

67. A. Strominger, C. Vafa, Phys. Lett. B 379, 99 (1996).

68. J.M. Maldacena, "Black Holes in String Theory", Ph.D. Thesis, Princeton University, hep-th/9607235.

69. T. Damour, "Quantum strings and black holes", in Proceedings of the 9th Marcel Grossmann Meeting, to appear, gr-qc/0104080.

70. A. Peet, "TASI lectures on black holes in string theory", hep-th/0105077.

71. J.P.S. Lemos, "Black Holes: from Galactic Nuclei to Elementary Particles", Proceedings of the XXI $\underline{t}^{h}$ Annual Meeting of the Brazilian Astronomical Society, eds. F. Jablonski, F. Elizalde, L. Sodré Jr., Vera Jablonsky, (Instituto Astronômico e Geofísico-USP, S. Paulo), p.57; astro-ph/9612220. 